\title{
Low abundance of mitofusin 2 in dairy cows with moderate fatty liver is associated with alterations in hepatic lipid metabolism
}

\author{
Jihong Dong, ${ }^{1}$ Juan J. Loor, ${ }^{2}$ Rankun Zuo, ${ }^{3}$ Xiying Chen, ${ }^{1}$ Yusheng Liang, ${ }^{2}$ Yazhe Wang, ${ }^{1}$ Xin Shu, ${ }^{1}$ \\ Xudong Sun, ${ }^{1}$ Hongdou Jia, ${ }^{1}$ Guowen Liu, ${ }^{1}$ Zhe Wang, ${ }^{1}$ Xiaobing Li, ${ }^{1}$ and Xinwei Li ${ }^{1 *}$ \\ ${ }^{1}$ Key Laboratory of Zoonosis Research, Ministry of Education, College of Veterinary Medicine, Jilin University, Changchun 130062, China \\ ${ }^{2}$ Mammalian NutriPhysioGenomics, Department of Animal Sciences and Division of Nutritional Sciences, University of Illinois, Urbana 61801 \\ ${ }^{3}$ College of Animal Science and Technology, Qingdao Agricultural University, Qingdao 266109, China
}

\section{ABSTRACT}

High blood concentrations of nonesterified fatty acids (NEFA) and altered lipid metabolism are key characteristics of fatty liver in dairy cows. In nonruminants, the mitochondrial membrane protein mitofusin 2 (MFN2) plays important roles in regulating mitochondrial function and intrahepatic lipid metabolism. Whether MFN2 is associated with hepatic lipid metabolism in dairy cows with moderate fatty liver is unknown. Therefore, to investigate changes in MFN2 expression and lipid metabolic status in dairy cows with moderate fatty liver, blood and liver samples were collected from healthy dairy cows $(\mathrm{n}=10)$ and cows with moderate fatty liver $(\mathrm{n}=10)$. To determine the effects of MFN2 on lipid metabolism in vitro, hepatocytes isolated from healthy calves were used for small interfering RNAmediated silencing of MFN2 or adenovirus-mediated overexpression of MFN2 for $48 \mathrm{~h}$, or treated with 0 , $0.6,1.2$, or $2.4 \mathrm{~m} M \mathrm{NEFA}$ for $12 \mathrm{~h}$. Milk production and plasma glucose concentrations in dairy cows with moderate fatty liver were lower, but concentrations of NEFA and $\beta$-hydroxybutyrate (BHB) were greater in dairy cows with moderate fatty liver. Dairy cows with moderate fatty liver displayed hepatic lipid accumulation and lower abundance of hepatic MFN2, peroxisome proliferator-activated receptor- $\alpha(P P A R \alpha)$, and carnitine palmitoyltransferase 1A (CPT1A). However, sterol regulatory element-binding protein 1c (SREBP1c), acetyl CoA carboxylase 1 (ACACA), fatty acid synthase (FASN), and diacylglycerol acyltransferase 1 (DGAT1) were more abundant in the livers of dairy cows with moderate fatty liver. In vitro, exogenous NEFA treatment upregulated abundance of SREBP1c, ACACA, FASN, and DGAT1, and downregulated the abundance of PPAR $\alpha$ and CPT1A. These changes

Received February 25, 2019.

Accepted April 18, 2019.

*Corresponding author: lixinwei100@126.com were associated with greater lipid accumulation in calf hepatocytes, and MFN2 silencing aggravated this effect. In contrast, overexpression of MFN2-ameliorated exogenous NEFA-induced lipid accumulation by downregulating the abundance of SREBP-1c, ACACA, FASN, and DGAT1, and upregulating the abundance of PPAR $\alpha$ and CPT1A in calf hepatocytes. Overall, these data suggest that one cause for the negative effect of excessive NEFA on hepatic lipid accumulation is the inhibition of MFN2. As such, these mechanisms partly explain the development of hepatic steatosis in dairy cows.

Key words: dairy cows, moderate fatty liver, mitofusin 2, lipid metabolism

\section{INTRODUCTION}

Fatty liver is a common disease in modern high-yielding dairy cows, occurring to varying degrees during the transition period (Hippen et al., 1999). High-producing dairy cows often suffer from negative energy balance during early lactation (Ingvartsen, 2006; Grummer, 2007), a state that is associated with fat mobilization, resulting in increased plasma concentrations of nonesterified fatty acids (NEFA; González et al., 2011). A high influx of NEFA into the liver results in excessive accumulation of triacylglycerol (TAG), which can cause fatty liver (Grummer, 1993). Approximately 30 to $40 \%$ of high-yielding dairy cows could be afflicted with moderate fatty liver in the first month after calving (Bobe et al., 2004). If untreated, severe fatty liver can develop and reduce production performance of cows, with eventual elimination from the herd. Therefore, prevention and treatment of moderate fatty liver is important for helping cows make uneventful transitions into lactation.

Mitochondria play an important role in the overall regulation of hepatic lipid metabolism (Sunny et al., 2017). Mitofusin 2 (MFN2), a transmembrane protein (Karbowski and Youle, 2003) located on the 
mitochondrial outer membrane (Rojo et al., 2002; Pich et al., 2005), promotes mitochondrial fusion, which is important for proper function of the mitochondrion. Gao et al. (2018) reported a marked hepatic mitochondrial dysfunction, along with decreased abundance of MFN2, in the livers of dairy cows with severe fatty liver. Whether this protein is associated with the development of fatty liver after calving remains unknown, especially in instances of moderate fatty liver.

High levels of NEFA, a pathological factor for fatty liver in dairy cows, are lipotoxic (Bobe et al., 2004) and could impair hepatic mitochondrial function (Gao et al., 2018). Accordingly, we hypothesized that a low expression of MFN2 mediates, at least in part, alterations in hepatic lipid metabolism induced by high concentrations of NEFA in dairy cows. Therefore, the aim of the current study was to investigate the role of MFN2 in lipid metabolism in cow hepatocytes.

\section{MATERIALS AND METHODS}

\section{Animals}

The protocol for the current study was approved by the Jilin University Ethics Committee on the Use and Care of Animals (Changchun, China; 2017 clinical trial 201711037). All cows for this study were selected from a 7,000-cow dairy farm located in Changchun City, Jilin Province, China. All cows underwent routine physical examinations to guarantee absence of other comorbidities. All cows received the same diet and were fed ad libitum (Supplemental Table S1; https://doi.org/10 .3168/jds.2019-16544). Based on hepatic TAG content (\% g/g of wet weight; Bobe et al., 2004), TAG content in healthy dairy cows was $<1 \%$, TAG content in dairy cows with mild fatty liver was 1 to $5 \%$, TAG content in dairy cows with moderate fatty liver was 5 to $10 \%$, and TAG content in dairy cows with severe fatty liver was $>10 \%$. The study used 10 healthy cows (hepatic TAG content $<1 \%$ ) and 10 cows with moderate fatty liver (hepatic TAG content 5 to 10\%). Cows had free access to clean water and were milked twice daily at 0530 and $1500 \mathrm{~h}$. The basic description of cows used in the study is provided in Table 1.

Blood samples were drawn via jugular venipuncture between 0700 and $0900 \mathrm{~h}$ on 3 consecutive days before feeding and immediately centrifuged at $4^{\circ} \mathrm{C}$ for 15 $\min$ at $3,500 \times g$. The serum obtained was stored at $-80^{\circ} \mathrm{C}$ until analysis. To obtain liver tissue samples, the intercostal space of cows was shaved, sanitized with iodine scrub and $75 \%$ alcohol, and anesthetized with subcutaneous injection of $2 \%$ lidocaine $\mathrm{HCl}$ (SigmaAldrich, St. Louis, MO). A scalpel blade was used to make a $3-\mathrm{mm}$ stab incision in the skin. The liver puncture needle (Shanghai Surgical Equipment Factory, Shanghai, China) was then inserted through the intercostal muscle layers and into the liver. Two liver tissue samples (approximately $150 \mathrm{mg}$ from each cow) were taken from the 11th or 12th right intercostal space and immediately frozen in liquid nitrogen or fixed with $10 \%$ formaldehyde neutral buffer solution. All cows were housed in a climate-controlled barn during this experiment.

\section{Determination of Blood Parameters}

The serum concentrations of glucose, NEFA, and BHB were determined using a Hitachi 7170 autoanalyzer (Hitachi, Tokyo, Japan) with commercially available kits (glucose: cat. no. GL3815; NEFA: cat. no. FA115; BHB: cat. no. RB1008; Randox Laboratories, Crumlin, $\mathrm{UK})$.

\section{Calf Hepatocyte Isolation and Culture}

Liver tissue was collected from 5 calves (age $1 \mathrm{~d}$, female, $30-40 \mathrm{~kg}$, healthy, fasting) for isolation of primary hepatocytes. Hepatocytes were isolated by a 2-step perfusion method as described by $\mathrm{Du}$ et al. (2018a). The 1-d-old calves were anesthetized, and

Table 1. Description of healthy cows (control; $\mathrm{n}=10)$ and cows with moderate fatty liver $(\mathrm{n}=10)$

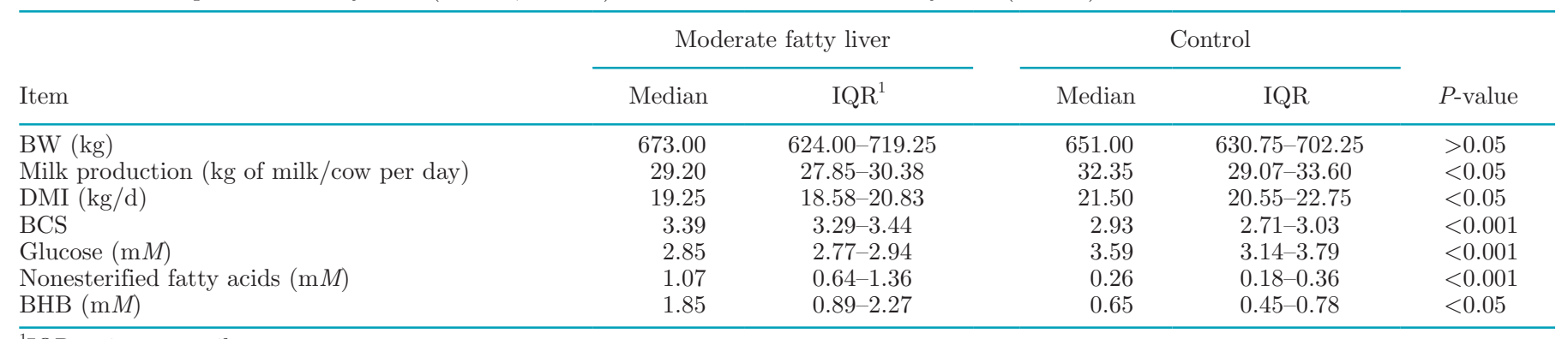

${ }^{1} \mathrm{IQR}=$ interquartile range. 
heparin sodium was administered intravenously. According to a previous method (Parker and Gaughan, 1988), the caudate lobe of each calf's liver was obtained by surgical excision. Subsequently, perfusion solution A $(140 \mathrm{~m} M \mathrm{NaCl}, 10 \mathrm{~m} M$ HEPES, $6.7 \mathrm{~m} M \mathrm{KCl}, 0.5 \mathrm{~m} M$ EDTA, and $2.5 \mathrm{~m} M$ glucose, $\mathrm{pH} 7.2-7.4,37^{\circ} \mathrm{C}$ ) was used to perfuse the caudate lobe of the liver at a flow rate of $50 \mathrm{~mL} / \mathrm{min}$ for $12 \mathrm{~min}$. Then, perfusion solution B $(140 \mathrm{~m} M \mathrm{NaCl}, 30 \mathrm{~m} M$ HEPES, $6.7 \mathrm{~m} M \mathrm{KCl}, 5 \mathrm{~m} M$ $\mathrm{CaCl}_{2}$, and $2.5 \mathrm{mM}$ glucose, $\mathrm{pH} 7.2-7.4,37^{\circ} \mathrm{C}$ ) was used to perfuse the caudate lobe of the liver at a flow rate of $50 \mathrm{~mL} / \mathrm{min}$ for approximately $3 \mathrm{~min}$, until the liquid became clear. Subsequently, perfusion solution C $(0.1 \mathrm{~g}$ collagenase IV dissolved in $0.5 \mathrm{~L}$ of perfusion solution $\mathrm{B}, \mathrm{pH} 7.2-7.4,37^{\circ} \mathrm{C}$ ) was used to perfuse the caudate lobe of the liver at a flow rate of $20 \mathrm{~mL} / \mathrm{min}$ for $15-20$ min. To terminate the collagenase digestion, $100 \mathrm{~mL}$ of fetal bovine serum (Hyclone Laboratories, Logan, UT) was added to the liver in a sterile flat plate, and the liver was cut into pieces with scissors. Incompletely digested tissue was discarded. Cell sieves of 100- (150 $\mu \mathrm{m})$ and 200 -mesh $(75 \mu \mathrm{m})$ were sequentially used to filter the liver parenchyma. After being washed twice in RPMI-1640 basic medium (Hyclone Laboratories), the hepatocyte suspension was centrifuged for 5 min at 500 $\times g$ at $4^{\circ} \mathrm{C}$. Isolated hepatocytes were transferred to 6 well plates at $1 \times 10^{6}$ cells $/ \mathrm{mL}$ and incubated at $37^{\circ} \mathrm{C}$ under $5 \% \mathrm{CO}_{2}$ in adherent medium (RPMI-1640 basic medium supplemented with $10 \%$ fetal bovine serum, $10^{-6} M$ insulin, $10^{-6} M$ dexamethasone, $10 \mu \mathrm{g} / \mathrm{mL}$ vitamin $\mathrm{C}$, and $1 \%$ penicillin/streptomycin). The adherent medium was replaced with growth medium containing $10 \%$ fetal bovine serum and $1 \%$ penicillin/streptomycin after $4 \mathrm{~h}$. The growth medium was replaced every $24 \mathrm{~h}$.

\section{Cell Treatments}

Cells were maintained in RPMI-1640 basic medium overnight before treating with NEFA. The medium was then replaced by RPMI-1640 basic medium with $2 \%$ BSA and treated with 0, 0.6, 1.2, and $2.4 \mathrm{mM}$ NEFA for $12 \mathrm{~h}$. The stock NEFA mix $(52.7 \mathrm{mM})$ solution (Du et al., 2018b; Jia et al., 2019) included oleic acid (22.9 $\mathrm{m} M)$, linoleic acid $(2.6 \mathrm{mM})$, palmitic acid $(16.8 \mathrm{mM})$, stearic acid $(7.6 \mathrm{mM})$, and palmitoleic acid $(2.8 \mathrm{mM}$; Sigma-Aldrich), and the $\mathrm{pH}$ of the NEFA solution was adjusted to 7.4 using hydrochloric acid $(1 \mathrm{M})$.

The hepatocytes were transfected with negative control of small interfering (si)RNA (Si-Control) or siRNA of MFN2 (Si-MFN2) with Lipofectamine 2000 (Invitrogen, Carlsbad, CA) in Opti-MEM I Reduced Serum Medium (Gibco, Waltham, MA) for $6 \mathrm{~h}$. The medium was then replaced with growth medium for $42 \mathrm{~h}$. The siRNA was designed and synthesized by
GenePharma (Shanghai, China) based on the bovine MFN2 mRNA sequence. The siRNA sequences are reported in Supplemental Table S2 (https://doi.org/10 .3168/jds.2019-16544).

The adenoviruses were constructed by Hanbio (Shanghai, China) and transfected into hepatocytes according to the manufacturer's instructions. Briefly, cells were transfected with empty adenovirus vectors (Ad-GFP) or MFN2 overexpression adenovirus (AdMFN2) according to the number of cells $\times$ multiplicity of infection/viral titers, and cultured with RPMI-1640 basic medium for $7 \mathrm{~h}$. Then, the medium was replaced with growth medium for $41 \mathrm{~h}$. The viral titers of AdGFP and Ad-MFN2 were $1.58 \times 10^{10}$ plaque-forming units $/ \mathrm{mL}$ and $1.26 \times 10^{10}$ plaque-forming units $/ \mathrm{mL}$, respectively, and the multiplicity of infection used in the present study was 50 .

\section{Liver Histology}

Liver tissue from $10 \%$ formaldehyde neutral buffer solution was embedded in paraffin and stained with hematoxylin/eosin after cutting to a thickness of $6 \mu \mathrm{m}$. For Oil Red O staining, bovine liver tissue and hepatocytes were frozen in an optimal cutting temperature compound (Sakura Finetek, Torrance, CA). The sections were sliced to $8 \mu \mathrm{m}$ on a freezing microtome at $-18^{\circ} \mathrm{C}$ and fixed in $75 \%$ alcohol at room temperature for $15 \mathrm{~min}$. The slides were stained using Oil Red O (O0625, Sigma-Aldrich) and then counterstained with hematoxylin.

Hepatocytes were washed twice in PBS and then fixed in $4 \%$ paraformaldehyde for $15 \mathrm{~min}$. Subsequently, hepatocytes were incubated in $60 \%$ isopropanol for 2 min and stained with $0.5 \%$ (wt/vol) Oil Red O solution for $30 \mathrm{~min}$. Then they were washed again with $60 \%$ isopropanol for $30 \mathrm{~s}$ and counterstained with hematoxylin before microscopy.

\section{TAG Content Determination}

Liver tissue (approximately $20 \mathrm{mg}$ ) was homogenized in 5\% Triton-X100 before being heated in a water bath $\left(85^{\circ} \mathrm{C}\right)$ for $3 \mathrm{~min}$. Then, liver tissue was vortexed and centrifuged at $2,000 \times g$ for $5 \mathrm{~min}$ at $4^{\circ} \mathrm{C}$. Supernatant was collected and stored at $-80^{\circ} \mathrm{C}$. Hepatocytes were collected with a cell scraper and washed twice with icecold PBS. The TAG content of liver tissue and hepatocytes was determined using an enzymatic kit (E1013; Applygen Technologies Inc., Beijing, China) according to the manufacturer's instructions. Total protein concentration was detected using the bicinchoninic acid method (P1511; Applygen Technologies Inc.). 


\section{Western Blotting}

Western blotting was performed as described by $\mathrm{Du}$ et al. (2017). Total protein was isolated from liver or hepatocytes using a commercial protein extraction kit (C510003; Sangon Biotech Co. Ltd., Shanghai, China). The protein concentrations of liver tissue and hepatocytes were detected using the bicinchoninic acid method. A total of $30 \mu \mathrm{g}$ of protein from each sample was separated by SDS-PAGE and transferred to 0.45$\mu \mathrm{m}$ PVDF membranes. The membranes were blocked with 3\% BSA Tris-buffered saline/Tween buffer solution for $4 \mathrm{~h}$ at room temperature, then incubated with MFN2 (1:1,000, ab56889; Abcam, Cambridge, UK), sterol regulatory element-binding protein 1c (SREBP-1c, 1:500, NB100-2215; Novus Biologicals, Centennial, CO), acetyl CoA carboxylase 1 (ACACA, 1:500; ab45174, Abcam), fatty acid synthase (FASN, 1:500; \#3180, Cell Signaling Technology Inc., Danvers, MA), diacylglycerol acyltransferase 1 (DGAT1, 1:500; ab54037, Abcam), peroxisome proliferator-activated receptor- $\alpha$ (PPAR $\alpha, 1: 1,000$; ab8934, Abcam), carnitine palmitoyltransferase 1A (CPT1A, 1:1,000; ab83862, Abcam), and $\beta$-actin (1:2000; ab8226, Abcam) antibodies overnight at $4^{\circ} \mathrm{C}$. Membranes were washed 3 times and incubated with horseradish peroxidaseconjugated anti-mouse or anti-rabbit antibody (Boster, Wuhan, China) for $45 \mathrm{~min}$ at room temperature. The protein bands were detected with an enhanced chemiluminescence solution (ECL, Millipore, Bedford, MA) and visualized using a protein imager (ProteinSimple, San Jose, CA). Last, all protein bands were analyzed using ImageJ software (Wayne Rasband, National Institutes of Health, Bethesda, MD).

\section{Quantitative Reverse-Transcription PCR Assay}

The quantitative reverse transcription PCR (qRTPCR) assay was performed as described by Jia et al. (2019). Total RNA was isolated from liver tissue and hepatocytes using RNAiso Plus (Takara Biotechnology Co. Ltd., Dalian, China) according to the manufacturer's instructions. The RNA concentration and quality were detected by calculating the ratio of UV activity at 260/280 nm using K5500 micro-spectrophotometer (Beijing Kaiao Technology Development Ltd., Beijing, China) and electrophoresis (1\% agarose gels). Then, 2 $\mu \mathrm{g}$ of total RNA was reverse transcribed into cDNA with a reverse transcription kit (Takara Biotechnology Co. Ltd.) according to the supplier's protocol. The 7500 Real-Time PCR System (Applied Biosystems, Waltham, MA) and SYBR green plus reagent kit (Roche, Basel, Switzerland) were used to determine the abundance of mRNA. The nucleotide sequences for genes were designed using Primer Express software (Applied Biosystems) according to NCBI bovine reference sequences. The conditions for qRT-PCR were as follows: initial denaturation at $94^{\circ} \mathrm{C}$ for $2 \mathrm{~min}$; followed by 35 cycles of amplification at $94^{\circ} \mathrm{C}$ for $10 \mathrm{~s}, 60^{\circ} \mathrm{C}$ for $15 \mathrm{~s}$, and $72^{\circ} \mathrm{C}$ for $30 \mathrm{~s}$; and extension at $72^{\circ} \mathrm{C}$ for 5 min. Internal controls were $A C T B$ and glyceraldehyde3-phosphate dehydrogenase (GAPDH; Morey et al., 2011; Du et al., 2018b; Supplemental Figure S1, https: //doi.org/10.3168/jds.2019-16544). The abundance of mRNA was normalized to $A C T B$ and $G A P D H$ and then quantified by the $2^{-\Delta \Delta C T}$ method (Wan et al., 2018). For in vivo qRT-PCR experiments, the reaction was performed in triplicate for each cow, and 10 cows per group were included. For in vitro qRT-PCR experiments, the reaction was performed 9 times for each group. The primers used for MFN2, ACTB, and GAPDH are shown in Supplemental Table S3 (https:// doi.org/10.3168/jds.2019-16544).

\section{Statistical Analysis}

All experiments were repeated at least 3 times. The results were analyzed using the SPSS 19.0 software package (IBM Corp., Armonk, NY). All data were tested for normality. A parametric statistical analysis was conducted using the paired $t$-test; comparisons among groups were calculated using one-way ANOVA with subsequent Bonferroni correction. All analyses were performed using GraphPad Prism 5.0 (GraphPad Software Inc., La Jolla, CA). Data were expressed as means \pm SEM; $P<0.05$ was defined as statistically significant, and $P<0.01$ was defined as statistically highly significant.

\section{RESULTS}

\section{Decreased MFN2 Expression and Altered Lipid Metabolism in Liver of Dairy Cows with Moderate Fatty Liver}

As shown in Table 1 , milk production $(P<0.05)$, DMI $(P<0.05)$, and blood concentration of glucose $(P$ $<0.001)$ were lower in dairy cows with moderate fatty liver. Conversely, the BCS $(P<0.001)$, blood concentrations of NEFA $(P<0.001)$, and BHB $(P<0.05)$ were greater in dairy cows with moderate fatty liver.

Hematoxylin-eosin revealed macrovesicular steatosis (Figure 1A). Oil Red O staining showed more lipid droplets (LD) in the livers of dairy cows with moderate fatty liver (Figure 1A). Furthermore, the hepatic TAG content in dairy cows with moderate fatty liver was 
greater $(P<0.01)$ than in healthy cows (Figure 1B). Moreover, mRNA and protein abundance of MFN2 were lower $(P<0.01, P<0.05)$ in the livers of dairy cows with moderate fatty liver than in those of healthy cows (Figure 1C, D, and E). The protein abundance of SREBP-1c, ACACA, FASN, and DGAT1 was greater
$(P<0.05, P<0.01)$ in the livers of dairy cows with moderate fatty liver than in those of healthy cows (Figure 1D and E). Conversely, the hepatic protein abundance of PPAR $\alpha$ and its target molecule CPT1A in dairy cows with moderate fatty liver was lower $(P<$ 0.05) than in healthy cows (Figure 1D and E).

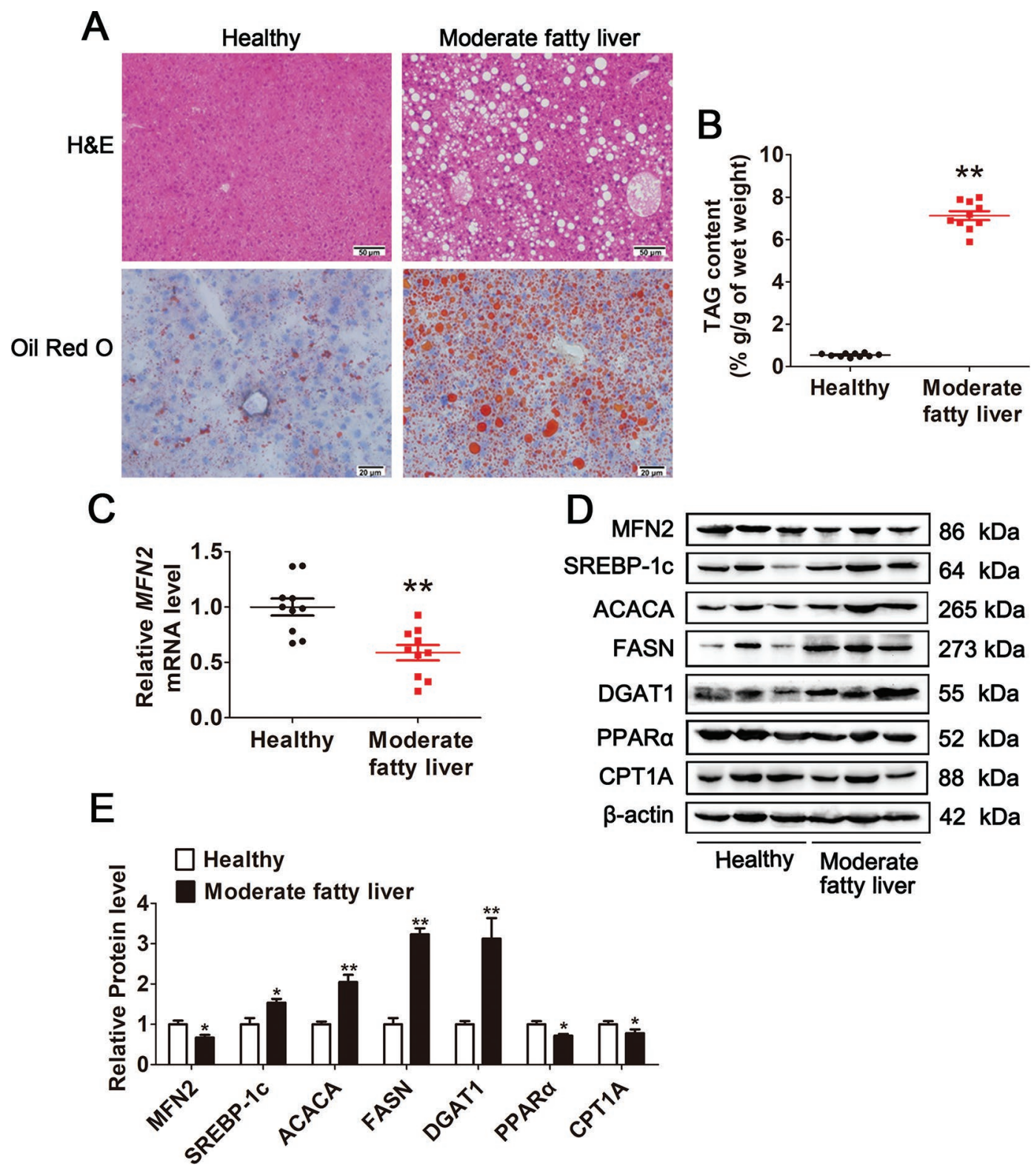

Figure 1. Dairy cows with moderate fatty liver exhibited altered lipid metabolism and decreased mitofusin 2 (MFN2) abundance. (A) Hematoxylin/eosin $(\mathrm{H} \& \mathrm{E}$; scale bar $=50 \mu \mathrm{m})$ and Oil Red O (scale bar $=20 \mu \mathrm{m})$ staining of liver sections. (B) Hepatic triacylglycerol (TAG) content in healthy dairy cows $(\mathrm{n}=10)$ and dairy cows with moderate fatty liver $(\mathrm{n}=10)$. (C) Relative mRNA expression levels of MFN2 in livers of healthy dairy cows $(\mathrm{n}=10)$ and dairy cows with moderate fatty liver $(\mathrm{n}=10)$. (D) Western blots analysis of MFN2, sterol regulatory element-binding protein 1c (SREBP-1c), acetyl CoA carboxylase 1 (ACACA), fatty acid synthase (FASN), diacylglycerol acyltransferase 1 (DGAT1), peroxisome proliferator-activated receptor- $\alpha$ (PPAR $\alpha$ ), and carnitine palmitoyltransferase 1A (CPT1A) in liver. (E) Relative protein expression levels of MFN2, SREBP-1c, ACACA, FASN, DGAT1, PPAR $\alpha$, and CPT1A in liver. The data of the healthy group were used to normalize the data of the moderate fatty liver group. Data were analyzed with paired $t$-test and expressed as the mean \pm SEM $(\mathrm{n}=10$ per group). ${ }^{*} P<0.05 ;{ }^{* *} P<0.01$. 
NEFA Promoted Lipid Synthesis and Inhibited the Abundance of MFN2 in Calf Hepatocytes

Oil Red $\mathrm{O}$ staining revealed that the amount and size of LD significantly increased with increasing NEFA concentrations (Figure 2A). Similarly, compared with controls, TAG content was greater $(P<0.01)$ in cells treated with 1.2 and $2.4 \mathrm{~m} M$ NEFA (Figure 2B). However, mRNA and protein abundance of MFN2 decreased as a function of increasing NEFA concentrations $(P<$ $0.01, P<0.05$; Figure $2 \mathrm{C}, \mathrm{D}$, and $\mathrm{E})$. The protein abundance of SREBP-1c, ACACA, FASN, and DGAT1 was greater $(P<0.05, P<0.01)$ in groups treated with $0.6,1.2$, and $2.4 \mathrm{~m} M$ NEFA (Figure $2 \mathrm{D}$ and E). However, the protein abundance of PPAR $\alpha$ and CPT1A was lower $(P<0.01)$ in groups treated with 1.2 and 2.4 $\mathrm{m} M$ NEFA (Figure 2D and E).

\section{MFN2 Silencing in Calf Hepatocytes Aggravates the Alterations in Lipid Metabolism \\ Induced by NEFA}

Oil Red $\mathrm{O}$ staining revealed that the amount and size of LD and the TAG content were greater in $\mathrm{Si}$ -

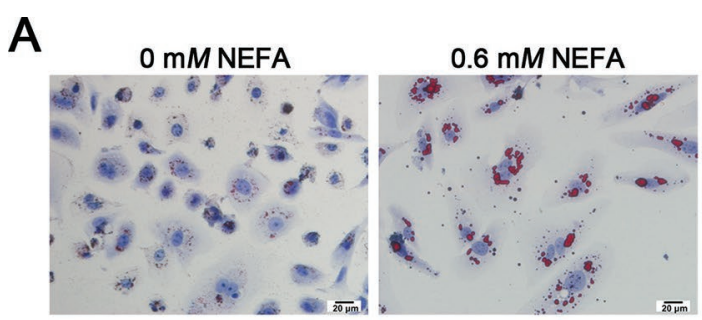

B

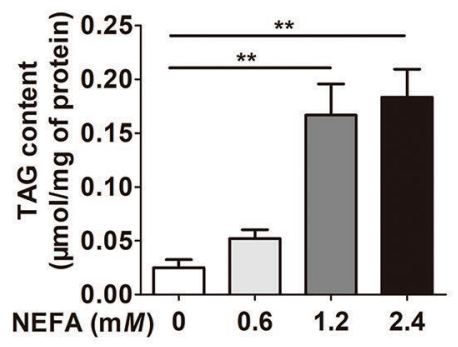

$1.2 \mathrm{mM}$ NEFA

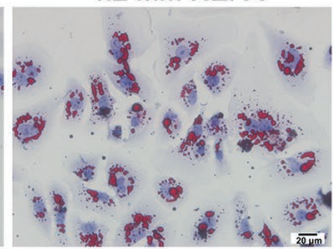

C

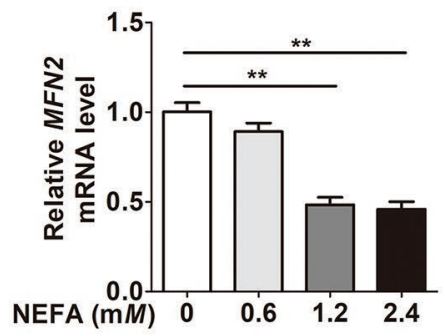

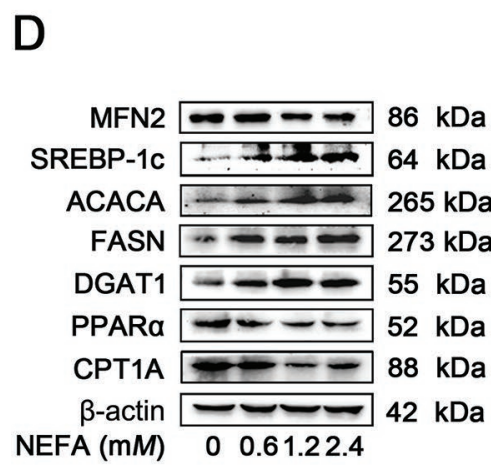

E $\square 0 \mathrm{~m} M$ NEFA $\quad \square 0.6 \mathrm{~m} M$ NEFA

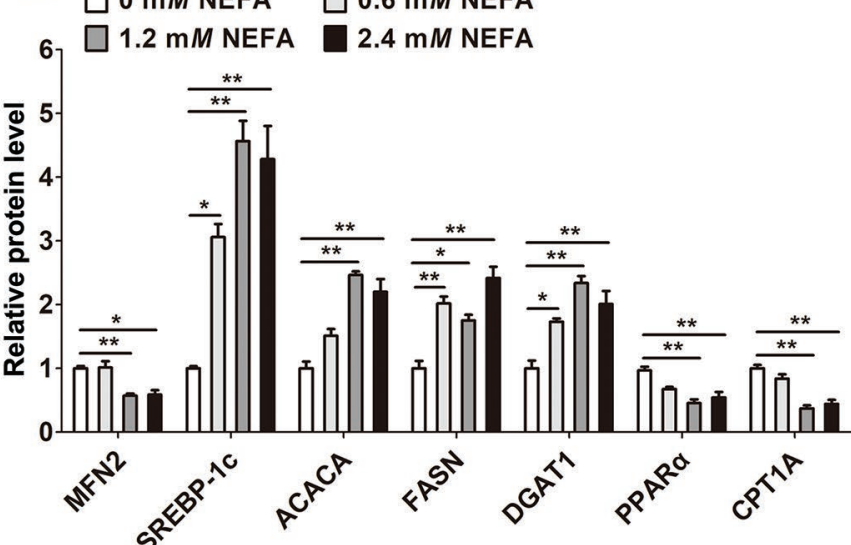

Figure 2. Nonesterified fatty acids (NEFA) inhibited the abundance of mitofusin 2 (MFN2) and induced altered lipid metabolism in calf hepatocytes. Calf hepatocytes were treated with 0, 0.6, 1.2, or $2.4 \mathrm{mM}$ NEFA and $2 \%$ BSA for $12 \mathrm{~h}$. (A) Oil Red O staining of calf hepatocytes; scale bar $=20 \mu \mathrm{m}$. (B) The triacylglycerol (TAG) content in calf hepatocytes. (C) Relative mRNA expression levels of MFN2. (D) Western blots analysis of MFN2, sterol regulatory element-binding protein 1c (SREBP-1c), acetyl CoA carboxylase 1 (ACACA), fatty acid synthase (FASN), diacylglycerol acyltransferase 1 (DGAT1), peroxisome proliferator-activated receptor- $\alpha$ (PPAR $\alpha$ ), and carnitine palmitoyltransferase 1A (CPT1A). (E) Relative protein expression levels of MFN2, SREBP-1c, ACACA, FASN, DGAT1, PPAR $\alpha$, and CPT1A. The data of the $0 \mathrm{~m} M$ NEFA group were used to normalize the data of each treatment group. Data were analyzed using a one-way ANOVA with subsequent Bonferroni correction and expressed as the mean \pm SEM. ${ }^{*} P<0.05 ;{ }^{*} P<0.01$. 
MFN2-treated groups than in Si-Control-treated groups $(P<0.01)$ and in Si-MFN2+1.2 $\mathrm{m} M$ NEFAtreated groups compared with Si-Control+1.2 $\mathrm{mM}$ NEFA-treated groups $(P<0.05$; Figure $3 \mathrm{~A}$ and $\mathrm{B})$. The mRNA and protein abundance of MFN2 was lower in the Si-MFN2-treated groups than in Si-Control $(P$ $<0.01)$ and in Si-MFN2+1.2 mM NEFA compared with Si-Control $+1.2 \mathrm{~m} M$ NEFA-treated groups $(P<$ $0.05, P<0.01$; Figure $3 \mathrm{C}, \mathrm{D}$, and $\mathrm{E})$. The protein abundance of SREBP-1c, ACACA, and DGAT1 was greater in Si-MFN2-treated groups than in Si-Controltreated groups $(P<0.01)$ and in Si-MFN2+1.2 $\mathrm{mM}$ NEFA-treated groups compared with $\mathrm{Si}$-Control+1.2 $\mathrm{m} M$ NEFA-treated groups $(P<0.01, P<0.05$; Figure $3 \mathrm{D}$ and $\mathrm{E}$ ). In addition, the protein abundance of FASN was greater $(P<0.05)$ in Si-MFN2-treated groups than in Si-Control-treated groups (Figure 3D and E). However, the protein abundance of PPAR $\alpha$ and CPT1A was lower in Si-MFN2-treated groups than in Si-Control-treated groups $(P<0.05, P<0.01)$ and in Si-MFN2+1.2 $\mathrm{m} M$ NEFA-treated groups compared with Si-Control+1.2 mM NEFA-treated groups $(P<$ 0.05; Figure 3D and E).

\section{MFN2 Overexpression in Calf Hepatocytes Alleviates Derangements in Lipid Metabolism Caused by NEFA}

Overexpression of MFN2 significantly improved lipid accumulation. The amount and size of LD (Figure 4A) and the TAG content (Figure 4B) were lower $(P<0.05)$ in Ad-MFN2+1.2 mM NEFA-treated groups than in Ad-GFP $+1.2 \mathrm{~m} M$ NEFA-treated groups. The mRNA and protein abundance of MFN2 was greater in AdMFN2-treated groups than in Ad-GFP-treated groups $(P<0.01)$ and in Ad-MFN2+1.2 NEFA-treated groups compared with Ad-GFP+1.2 NEFA-treated groups $(P<0.01$; Figure $4 \mathrm{C}, \mathrm{D}$, and $\mathrm{E})$. The protein abundance of SREBP-1c, ACACA, and DGAT1 was lower in Ad-MFN2-treated groups than in Ad-GFP treated groups $(P<0.05, P<0.01)$, and Ad-MFN2+1.2 $\mathrm{mM}$ NEFA-treated groups compared with Ad-GFP+1.2 $\mathrm{m} M$ NEFA-treated groups $(P<0.01$; Figure $4 \mathrm{D}$ and E). Furthermore, the protein abundance of FASN was lower $(P<0.01)$ in Ad-MFN2+1.2 m $M$ NEFA-treated groups compared with Ad-GFP+1.2 $\mathrm{m} M$ NEFA-treated groups (Figure 4D and E). However, the protein abundance of PPAR $\alpha$ was greater $(P<0.05)$ in AdMFN2-treated groups than in Ad-GFP-treated groups, and the protein abundance of PPAR $\alpha$ and CPT1A was greater $(P<0.01, P<0.05)$ in Ad-MFN2+1.2 $\mathrm{mM}$ NEFA-treated groups compared with Ad-GFP +1.2 $\mathrm{m} M$ NEFA-treated groups (Figure 4D and E).

\section{DISCUSSION}

Severe negative energy balance-induced fatty acid release at the beginning of lactation is one of the pathological bases of fatty liver development in cows (Li et al., 2014). Overloaded fatty acids are re-esterified and stored as TAG in hepatocytes, which eventually results in fatty liver (Jia et al., 2019; Zang et al., 2019). Mitochondria are essential organelles for hepatocytes to maintain lipid homeostasis and regulation of lipid metabolism (Koliaki et al., 2015). Importantly, the regulation and maintenance of mitochondrial function requires regulators such as MFN2, which play critical roles in regulating mitochondria respiration and fusion, as well as in regulation of lipid and glucose metabolism (Bach et al., 2003; Pich et al., 2005). Results from Gao et al. (2018) showed that the hepatic protein level of MFN2 was decreased in dairy cows with severe hepatic steatosis. Furthermore, high levels of NEFA decreased the abundance of MFN2. In this study, we confirmed the lower mRNA of MFN2 and also detected lower protein abundance in dairy cows with moderate fatty liver. Because the degree of downregulated abundance of MFN2 in cows with moderate fatty liver was lower than those with severe fatty liver (Gao et al., 2018), the data strongly suggest that abundance of MFN2 is closely associated with the degree of hepatic steatosis. Furthermore, the in vitro data reveal that excessive NEFA influx into the liver inhibits MFN2 and may be one factor contributing to increased TAG accumulation in hepatocytes.

Hepatic steatosis in dairy cows results primarily from the imbalance of fatty acid oxidation, synthesis of TAG, and export of TAG in very low density lipoproteins. In nonruminants, hepatic TAG accumulation is strongly associated with transcriptional activity and abundance of SREBP-1c, in part because this protein regulates genes that are involved in fatty acid and TAG synthesis, including ACACA, FASN, and DGAT1 (Porstmann et al., 2008; Villanueva et al., 2009). Our previous (Jia et al., 2019) and present studies revealed that the abundance of SREBP-1c and its target molecules ACACA, FASN, and DGAT1 was overinduced in the livers of dairy cows with moderate fatty liver. Furthermore, treatment of calf primary hepatocytes with high concentrations of fatty acids upregulated the expression of lipogenic molecules (ACACA, FASN, and DGAT1), in part by increasing the expression and activity of SREBP-1c. These data suggest that lipogenesis is one component of moderate fatty liver development in cows.

At least in nonruminants, PPAR $\alpha$ plays an important role in hepatic lipid metabolism via the induction of several enzymes of fatty acid $\beta$-oxidation, including 


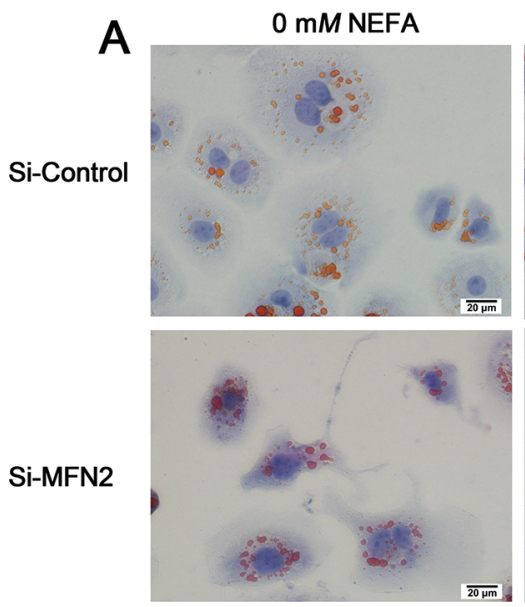

C

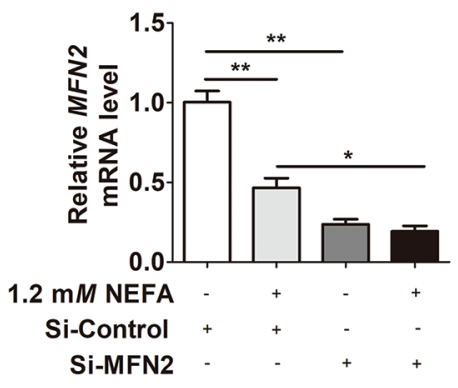

$\mathrm{E}$

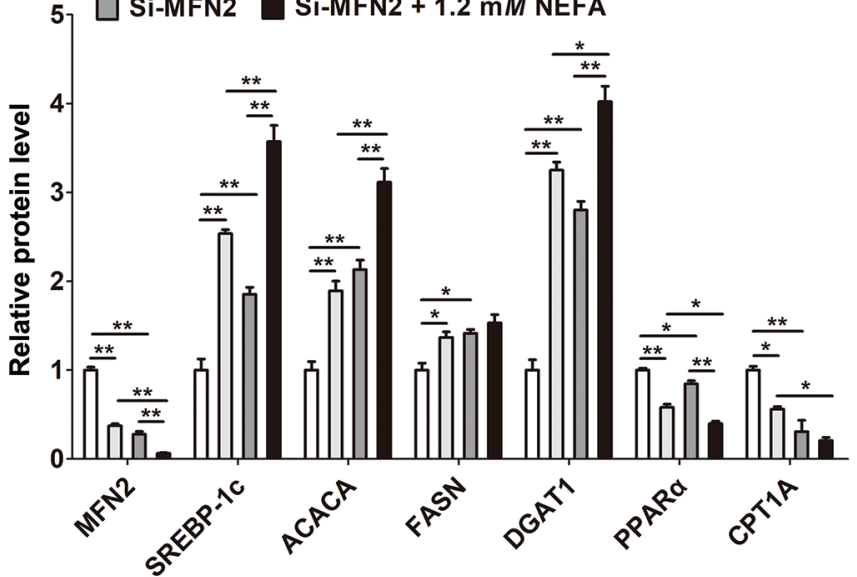

B

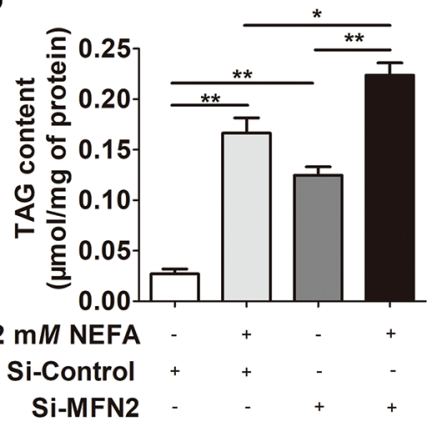

Figure 3. Mitofusin 2 (MFN2) silencing promoted altered lipid metabolism caused by exogenous nonesterified fatty acids (NEFA) in calf hepatocytes. Calf hepatocytes were divided into 4 groups as follows: (1) Si-Control group (cells were transfected with control of small interfering (si)RNA for $48 \mathrm{~h}$ and incubated with $2 \%$ BSA for $12 \mathrm{~h}$ ); (2) Si-Control+1.2 mM NEFA group (cells were transfected with control of siRNA for $48 \mathrm{~h}$ and treated with $1.2 \mathrm{mM}$ NEFA and 2\% BSA for $12 \mathrm{~h}$ ); (3) Si-MFN2 group (cells were transfected with silencing of MFN2 siRNA for $48 \mathrm{~h}$ and incubated with $2 \%$ BSA for $12 \mathrm{~h}$ ); and (4) Si-MFN2+1.2 mM NEFA group (cells were transfected with silencing of MFN2 siRNA for $48 \mathrm{~h}$ and treated with $1.2 \mathrm{mM}$ NEFA and $2 \%$ BSA for $12 \mathrm{~h}$ ). Treatment of Si-Control had no effect on hepatocytes' lipid metabolism. (A) Oil Red O staining of calf hepatocytes; scale bar $=20 \mu \mathrm{m}$. (B) The triacylglycerol (TAG) content in calf hepatocytes. (C) Relative mRNA expression levels of MFN2. (D) Western blot analysis of MFN2, sterol regulatory element-binding protein 1c (SREBP-1c), acetyl CoA carboxylase 1 (ACACA), fatty acid synthase (FASN), diacylglycerol acyltransferase 1 (DGAT1), peroxisome proliferator-activated receptor- $\alpha$ (PPAR $\alpha$ ), and carnitine palmitoyltransferase 1A (CPT1A). (E) Relative protein expression levels of MFN2, SREBP-1c, ACACA, FASN, DGAT1, PPAR $\alpha$, and CPT1A The data of the Si-Control group were used to normalize the data of each treatment group. Data were analyzed using a one-way ANOVA with subsequent Bonferroni correction and expressed as the mean \pm SEM. ${ }^{*} P<0.05 ;{ }^{* *} P<0.01$. 


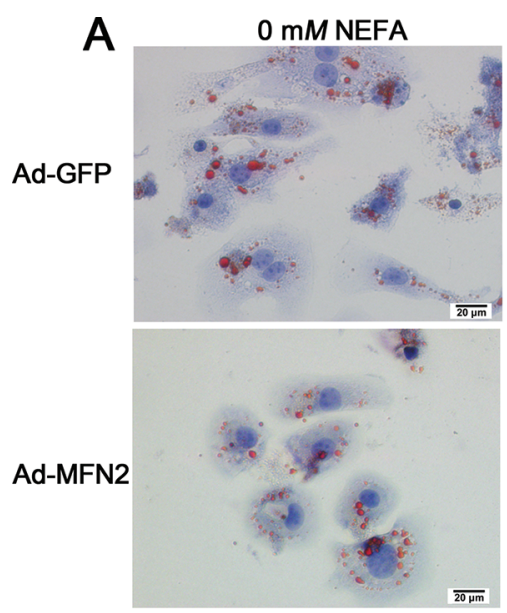

C

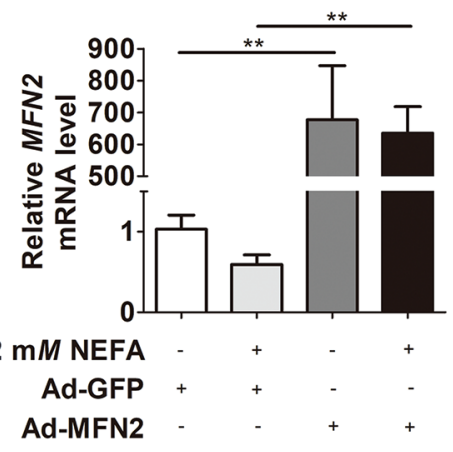

$1.2 \mathrm{mM}$ NEFA

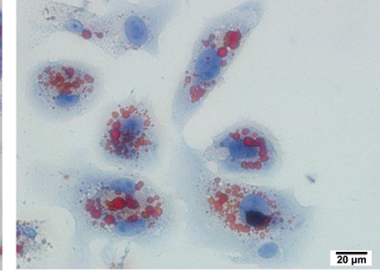

$\overline{20 \mu m}$

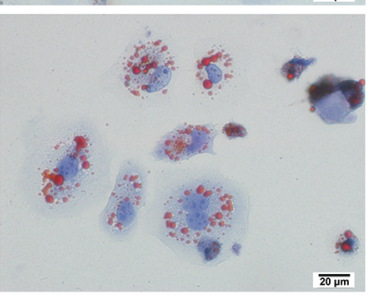

B

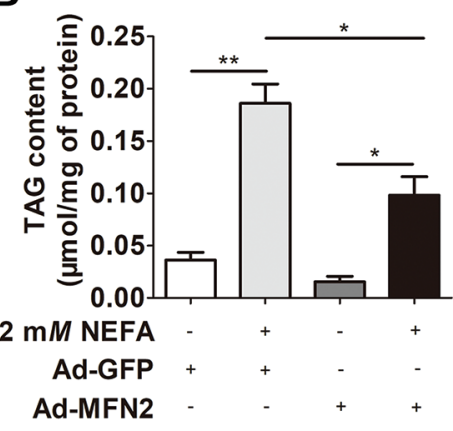

E

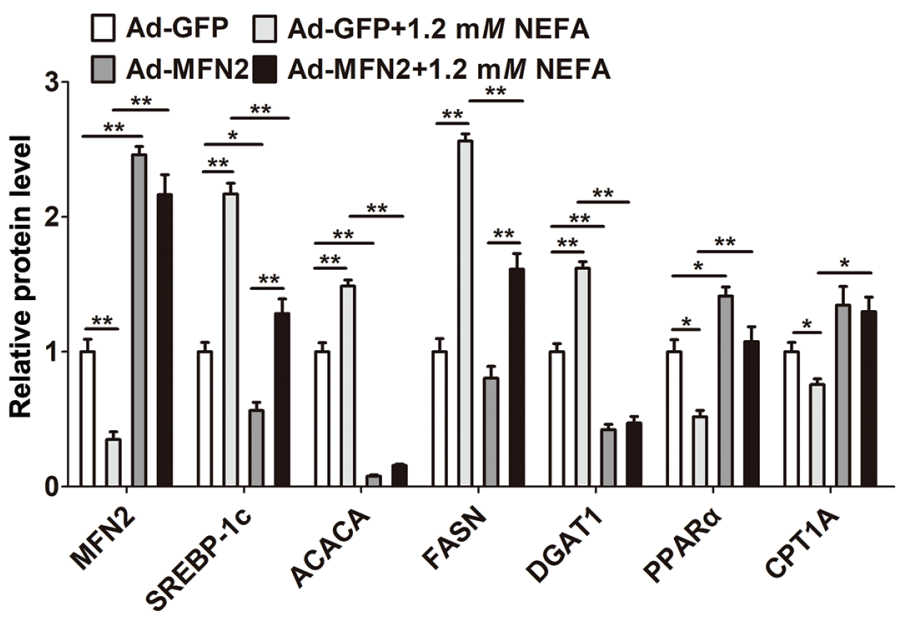

Figure 4. Mitofusin 2 (MFN2) overexpression alleviated altered lipid metabolism caused by exogenous nonesterified fatty acids (NEFA) in calf hepatocytes. Calf hepatocytes were divided into 4 groups as follows: (1) Ad-GFP group (cells were transfected with empty adenovirus vector for $48 \mathrm{~h}$ and incubated with $2 \%$ BSA for $12 \mathrm{~h}$ ); (2) Ad-GFP+1.2 mM NEFA group (cells were transfected with empty adenovirus vector for $48 \mathrm{~h}$ and treated with $1.2 \mathrm{mM}$ NEFA and 2\% BSA for $12 \mathrm{~h}$ ); (3) Ad-MFN2 group (cells were transfected with MFN2 overexpression adenovirus for 48 $\mathrm{h}$ and incubated with $2 \%$ BSA for $12 \mathrm{~h}$ ); and (4) Ad-MFN2+1.2 mM NEFA group (cells were transfected with MFN2 overexpression adenovirus for $48 \mathrm{~h}$ and treated with $1.2 \mathrm{mM}$ NEFA and $2 \%$ BSA for $12 \mathrm{~h}$ ). Treatment of Ad-GFP had no effect on hepatocytes' lipid metabolism. (A) Oil Red O staining of calf hepatocytes; scale bar $=20 \mu \mathrm{m}$. (B) The triacylglycerol (TAG) content in calf hepatocytes. (C) Relative mRNA expression levels of MFN2. (D) Western blots analysis of MFN2, sterol regulatory element-binding protein 1c (SREBP-1c), acetyl CoA carboxylase 1 (ACACA), fatty acid synthase (FASN), diacylglycerol acyltransferase 1 (DGAT1), peroxisome proliferator-activated receptor- $\alpha$ (PPAR $\alpha$ ), and carnitine palmitoyltransferase 1A (CPT1A). (E) Relative protein expression levels of MFN2, SREBP-1c, ACACA, FASN, DGAT1, PPAR , and CPT1A. The data of the Ad-GFP group were used to normalize the data of each treatment group. Data were analyzed using a one-way ANOVA with subsequent Bonferroni correction and expressed as the mean \pm SEM. ${ }^{*} P<0.05 ; * * P<0.01$. 
CPT1 and carnitine palmitoyltransferase 2 (CPT2; Li et al., 2013). The role of this nuclear receptor in mice was demonstrated 20 years ago, when knockdown of PPAR $\alpha$ was shown to lead to hepatic steatosis, hypoglycemia, and hypoketonemia (Leone et al., 1999). The lower abundance of PPAR $\alpha$ and its target molecule CPT1A in cases of moderate fatty liver, coupled with in vitro data demonstrating that high levels of fatty acids impaired the expression of PPAR $\alpha$ and CPT1A, suggested that suppression of fatty acid oxidation is a cause of TAG accumulation in dairy cows with moderate fatty liver. Thus, along with an apparent activation of the lipogenic program during severe negative energy balance, high concentrations of NEFA and decreased fatty acid oxidation contribute to hepatic steatosis during both moderate and severe instances of clinical disease (Gao et al., 2018; Jia et al., 2019).

Studies in humans and mice demonstrated that hepatic steatosis was associated with low expression of MFN2 (Galloway et al., 2014; Gao et al., 2018). In addition, mice subjected to a high-fat diet had lower hepatic MFN2 expression, in parallel with insulin resistance and hepatic steatosis (Gan et al., 2013). In highly oxidative tissues such as muscle, repression of MFN2 leads to a decrease in oxygen consumption, glucose and fatty acid oxidation, and mitochondrial membrane potential (Bach et al., 2003; Pich et al., 2005). Furthermore, deficiency of MFN2 causes mitochondrial dysfunction and defective insulin signaling in mouse livers (Sebastián et al., 2012). Thus, together with previous data from cows with severe fatty liver (Gao et al., 2018), the present data underscore that low abundance of MFN2 mediates, at least in part, the alterations in lipid metabolism in cases of moderate fatty liver.

Additional support for a biologic role of MFN2 and the onset of fatty liver can be discerned through the accumulation of TAG in hepatocytes upon silencing of MFN2 by siRNA. More importantly, the absence of MFN2 activity and the high concentrations of NEFA induced the lipogenic program via upregulation of SREBP-1c and its target molecules ACACA, FASN, and DGAT1, coupled with downregulation of PPAR $\alpha$ and CPT1A expression. Thus, continuous exposure of hepatocytes to a high content of NEFA can not only induce lipotoxicity and impair hepatic expression of MFN2 but also can clearly aggravate the altered lipid metabolic state by eliciting a lipogenic response.

In rat livers, overexpression of MFN2 through injection of adenovirus reversed insulin resistance induced by feeding a high-fat diet, via targeting the insulin signaling pathway, which was evidenced by the increased expression of insulin receptor (INSR), insulin receptor substrate 2 (IRS2), glucose transporter type 2 (GLUT2), and the phosphorylation levels of phosphoinositide3-kinase (PI3K) and protein kinase $\beta$ (AKT2; Gan et al., 2013). In cow hepatocytes, overexpression of MFN2 attenuated insulin resistance induced by high levels of NEFA (Gao et al., 2018). These studies suggest that MFN2 plays a positive role in hepatic lipid metabolism.

In the present study, the decrease in abundance of lipogenic molecules (SREBP-1c, ACACA, FASN, and DGAT1), coupled with upregulation of fatty acid oxidation molecules (PPAR $\alpha$ and CPT1A), upon adenoviral transfection of MFN2, suggests that upregulation of MFN2 can attenuate the negative effects on lipid metabolism induced by high circulating NEFA. This idea is supported by previous data demonstrating that MFN2 overexpression increased CPT1 expression in rat skeletal muscle (Zhang et al., 2013). That effect enhanced transport of NEFA into mitochondria for $\beta$-oxidation, thereby promoting fatty acid utilization. Together, the data indicate that MFN2 might serve as a useful therapeutic target for prevention and treatment of fatty liver in dairy cows.

In the transition period, fatty liver and ketosis are common diseases among dairy cows. These diseases can cause huge economic losses as a result of longer calving intervals, decreased milk production, shorter average lifespans of cows, and increased veterinary costs (Bobe et al., 2004). However, the underlying causes and mechanisms behind fatty liver and ketosis are unclear. Our research group focuses on the molecular mechanisms of fatty liver and ketosis from different aspects. In previous studies, Du et al. (2018a) illustrated the hepatic mitochondria adaptive mechanism in dairy cows with mild fatty liver. Jia et al. (2019) demonstrated that NEFA treatment could upregulate perilipin 5 expression and further induced TAG accumulation and inhibited the very low density lipoprotein assembly in hepatocytes. Du et al. (2018b) also found that the hepatic growth hormone-insulin-like growth factor-I axis was impaired in ketotic cows. In the present study, we demonstrated that MFN2 plays vital roles in intrahepatic lipid metabolism and that its low expression is involved in fatty liver development. These studies have partly illustrated the molecular pathogenesis of fatty liver and ketosis. However, the pathology and etiology of fatty liver and ketosis in dairy cows are complicated. Therefore, more studies are needed to elucidate the pathogenesis mechanism of fatty liver and ketosis and to further identify effective therapeutic strategies.

\section{CONCLUSIONS}

Dairy cows with moderate fatty liver display hepatic steatosis and lower abundance of MFN2. In vitro data 
indicate that high levels of NEFA inhibit MFN2 expression, leading to upregulation of lipid synthesis molecules (SREBP-1c, ACACA, FASN, and DGAT1) and inhibition of lipid oxidation molecules (PPAR $\alpha$ and CPT1A). Those events lead to TAG accumulation in hepatocytes. Thus, alterations in MFN2 expression clearly influence susceptibility to fatty liver development during the postpartum period. Mitofusin 2 protein may be a novel target in the treatment of dairy cows with moderate fatty liver during the perinatal period.

\section{ACKNOWLEDGMENTS}

This work was supported by the National Key Research and Development Program (Beijing, China; grant no. 2016YFD0501206), the National Natural Science Foundation of China (Beijing, China; grant nos. 31772810, 3167262, and 315725811), the Jilin Natural Science Foundation (Changchun, China; grant no. 20170101148JC), the Natural Science Scientific Research Project Jointly Established by Jilin University and Jilin Province (Changchun, China; grant no. SXGJSF2017-6), and the Talents Cultivation Program of Jilin University. The authors declare no conflicts of interest.

\section{REFERENCES}

Bach, D., S. Pich, F. X. Soriano, N. Vega, B. Baumgartner, J. Oriola, J. R. Daugaard, J. Lloberas, M. Camps, J. R. Zierath, R. RabasaLhoret, H. Wallberg-Henriksson, M. Laville, M. Palacin, H. Vidal, F. Rivera, M. Brand, and A. Zorzano. 2003. Mitofusin-2 determines mitochondrial network architecture and mitochondrial metabolism. A novel regulatory mechanism altered in obesity. J. Biol. Chem. 278:17190-17197.

Bobe, G., J. W. Young, and D. C. Beitz. 2004. Invited review: Pathology, etiology, prevention, and treatment of fatty liver in dairy cows. J. Dairy Sci. 87:3105-3124.

Du, X., T. Shen, H. Wang, X. Qin, D. Xing, Q. Ye, Z. Shi, Z. Fang, Y. Zhu, Y. Yang, Z. Peng, C. Zhao, B. Lv, X. Li, G. Liu, and X. Li. 2018a. Adaptations of hepatic lipid metabolism and mitochondria in dairy cows with mild fatty liver. J. Dairy Sci. 101:9544-9558.

Du, X., Z. Shi, Z. Peng, C. Zhao, Y. Zhang, Z. Wang, X. Li, G. Liu, and X. Li. 2017. Acetoacetate induces hepatocytes apoptosis by the ROS-mediated MAPKs pathway in ketotic cows. J. Cell. Physiol. 232:3296-3308.

Du, X., Y. Zhu, Z. Peng, Y. Cui, Q. Zhang, Z. Shi, Y. Guan, X. Sha, T. Shen, Y. Yang, X. Li, Z. Wang, X. Li, and G. Liu. 2018b. High concentrations of fatty acids and beta-hydroxybutyrate impair the growth hormone-mediated hepatic JAK2-STAT5 pathway in clinically ketotic cows. J. Dairy Sci. 101:3476-3487.

Galloway, C. A., H. Lee, P. S. Brookes, and Y. Yoon. 2014. Decreasing mitochondrial fission alleviates hepatic steatosis in a murine model of nonalcoholic fatty liver disease. Am. J. Physiol. Gastrointest. Liver Physiol. 307:G632-G641.

Gan, K. X., C. Wang, J. H. Chen, C. J. Zhu, and G. Y. Song. 2013. Mitofusin-2 ameliorates high-fat diet-induced insulin resistance in liver of rats. World J. Gastroenterol. 19:1572-1581.

Gao, W., X. Du, L. Lei, H. Wang, M. Zhang, Z. Wang, X. Li, G. Liu, and X. Li. 2018. NEFA-induced ROS impaired insulin signalling through the JNK and p38MAPK pathways in non-alcoholic steatohepatitis. J. Cell. Mol. Med. 22:3408-3422.

González, F. D., R. Muino, V. Pereira, R. Campos, and J. L. Benedito. 2011. Relationship among blood indicators of lipomobilization and hepatic function during early lactation in high-yielding dairy cows. J. Vet. Sci. 12:251-255.

Grummer, R. R. 1993. Etiology of lipid-related metabolic disorders in periparturient dairy cows. J. Dairy Sci. 76:3882-3896.

Grummer, R. R. 2007. Strategies to improve fertility of high yielding dairy farms: Management of the dry period. Theriogenology 68(Suppl. 1):S281-S288

Hippen, A. R., P. She, J. W. Young, D. C. Beitz, G. L. Lindberg, L. F. Richardson, and R. W. Tucker. 1999. Alleviation of fatty liver in dairy cows with 14-day intravenous infusions of glucagon. J. Dairy Sci. 82:1139-1152.

Ingvartsen, K. L. 2006. Feeding- and management-related diseases in the transition cow: Physiological adaptations around calving and strategies to reduce feeding-related diseases. Anim. Feed Sci. Technol. 126:175-213.

Jia, H., X. Li, G. Liu, J. J. Loor, R. Buctrout, X. Sun, G. Li, X. Shu, J. Dong, Y. Wang, R. Zuo, Z. Wang, and X. Li. 2019. Perilipin 5 promotes hepatic steatosis in dairy cows through increasing lipid synthesis and decreasing very low density lipoprotein assembly. J. Dairy Sci. 102:833-845.

Karbowski, M., and R. J. Youle. 2003. Dynamics of mitochondrial morphology in healthy cells and during apoptosis. Cell Death Differ. 10:870-880.

Koliaki, C., J. Szendroedi, K. Kaul, T. Jelenik, P. Nowotny, F. Jankowiak, C. Herder, M. Carstensen, M. Krausch, W. T. Knoefel, M. Schlensak, and M. Roden. 2015. Adaptation of hepatic mitochondrial function in humans with non-alcoholic fatty liver is lost in steatohepatitis. Cell Metab. 21:739-746.

Leone, T. C., C. J. Weinheimer, and D. P. Kelly. 1999. A critical role for the peroxisome proliferator-activated receptor alpha (PPARalpha) in the cellular fasting response: The PPARalpha-null mouse as a model of fatty acid oxidation disorders. Proc. Natl. Acad. Sci. USA 96:7473-7478.

Li, X., H. Chen, Y. Guan, X. Li, L. Lei, J. Liu, L. Yin, G. Liu, and Z. Wang. 2013. Acetic acid activates the AMP-activated protein kinase signaling pathway to regulate lipid metabolism in bovine hepatocytes. PLoS One 8:e67880.

Li, X., Y. Li, W. Yang, C. Xiao, S. Fu, Q. Deng, H. Ding, Z. Wang, G. Liu, and X. Li. 2014. SREBP-1c overexpression induces triglycerides accumulation through increasing lipid synthesis and decreasing lipid oxidation and VLDL assembly in bovine hepatocytes. J. Steroid Biochem. Mol. Biol. 143:174-182.

Morey, S. D., L. K. Mamedova, D. E. Anderson, C. K. Armendariz, E. C. Titgemeyer, and B. J. Bradford. 2011. Effects of encapsulated niacin on metabolism and production of periparturient dairy cows. J. Dairy Sci. 94:5090-5104.

Parker, J. E., and E. M. Gaughan. 1988. Partial hepatic resection for treatment of a single liver abscess in a dairy heifer. Vet. Surg. 17:87-89.

Pich, S., D. Bach, P. Briones, M. Liesa, M. Camps, X. Testar, M. Palacin, and A. Zorzano. 2005. The Charcot-Marie-Tooth type 2A gene product, Mfn2, up-regulates fuel oxidation through expression of OXPHOS system. Hum. Mol. Genet. 14:1405-1415.

Porstmann, T., C. R. Santos, B. Griffiths, M. Cully, M. Wu, S. Leevers, J. R. Griffiths, Y. L. Chung, and A. Schulze. 2008. SREBP activity is regulated by mTORC1 and contributes to Akt-dependent cell growth. Cell Metab. 8:224-236.

Rojo, M., F. Legros, D. Chateau, and A. Lombes. 2002. Membrane topology and mitochondrial targeting of mitofusins, ubiquitous mammalian homologs of the transmembrane GTPase Fzo. J. Cell Sci. 115:1663-1674.

Sebastián, D., M. I. Hernández-Alvarez, J. Segalés, E. Sorianello, J. P. Muñoz, D. Sala, A. Waget, M. Liesa, J. C. Paz, P. Gopalacharyulu, M. Orešič, S. Pich, R. Burcelin, M. Palacín, and A. Zorzano. 2012. Mitofusin 2 (Mfn2) links mitochondrial and endoplasmic reticulum 
function with insulin signaling and is essential for normal glucose homeostasis. Proc. Natl. Acad. Sci. USA 109:5523-5528.

Sunny, N. E., F. Bril, and K. Cusi. 2017. Mitochondrial adaptation in nonalcoholic fatty liver disease: Novel mechanisms and treatment strategies. Trends Endocrinol. Metab. 28:250-260.

Villanueva, C. J., M. Monetti, M. Shih, P. Zhou, S. M. Watkins, S. Bhanot, and R. V. Farese Jr.. 2009. Specific role for acyl CoA: Diacylglycerol acyltransferase 1 (Dgat1) in hepatic steatosis due to exogenous fatty acids. Hepatology 50:434-442.

Wan, D., Y. Zhang, X. Wu, X. Lin, X. Shu, X. Zhou, H. Du, W. Xing, H. Liu, L. Li, Y. Li, and Y. Yin. 2018. Maternal dietary supplementation with ferrous $\mathrm{N}$-carbamylglycinate chelate affects sow reproductive performance and iron status of neonatal piglets. Animal 12:1372-1379.

Zang, Y., S. S. Samii, W. A. Myers, H. R. Bailey, A. N. Davis, E. Grilli, and J. W. McFadden. 2019. Methyl donor supplementation suppresses the progression of liver lipid accumulation while modifying the plasma triacylglycerol lipidome in periparturient Holstein dairy cows. J. Dairy Sci. 102:1224-1236.

Zhang, X., C. Wang, G. Song, K. Gan, D. Kong, Q. Nie, and L. Ren. 2013. Mitofusion-2-mediated alleviation of insulin resistance in rats through reduction in lipid intermediate accumulation in skeletal muscle. J. Biomed. Sci. 20:45. 